\title{
Residual Set Up Errors of the Surrogate-guided Registration Using Four-dimensional CT Images and Breath Holding Ones in Respiratory Gated Radiotherapy for Liver Cancer
}

\author{
YOSHIHIRO UEDA ${ }^{1}$, MARI TSUJII ${ }^{1}$, SHINGO OHIRA $^{1}$, IORI SUMIDA ${ }^{2}$, \\ MASAYOSHI MIYAZAKI $^{1}$ and TERUKI TESHIMA ${ }^{1}$ \\ ${ }^{1}$ Department of Radiation Oncology, Osaka International Cancer Institute, Osaka, Japan; \\ ${ }^{2}$ Department of Radiation Oncology, Osaka University Graduate School of Medicine, Osaka, Japan
}

\begin{abstract}
Background/Aim: To evaluate the surrogateguided registration accuracy of two computed tomography (CT) image sets, expiratory phase four-dimensional (Ex4D) $C T$ and breath-holding $C T$ (BHCT), in respiratory-gated radiotherapy for liver cancer. Materials and Methods: The surrogate-guided registration errors were defined as the differences between the diaphragm-and fiducial-guided registrations or the differences between upper and lower fiducial registrations in three directions: left-right $(L R)$, anterior-posterior (AP), and cranio-caudal (CC). Results: The mean $\pm S D$ s of the absolute errors for diaphragm-guided registration were $1.9 \pm 1.3,2.7 \pm 1.8$, and $2.6 \pm 1.7 \mathrm{~mm}$ with Ex4D and 1.8 $\pm 1.8,2.6 \pm 1.9$, and $1.8 \pm 1.7 \mathrm{~mm}$ with $B H C T$ in the LR, AP and CC directions, respectively (CC direction, $p<0.01)$. In the fiducial-guided registration, there were no significant differences in any direction. In registration with Ex4D, there were positive correlations between registration errors and the respiratory irregularity during $4 D$ scanning (correlation coefficient; diaphragm: 0.65, fiducial: 0.54). Conclusion: BHCT has the advantage of accurate surrogateguided registration compared with Ex4D.
\end{abstract}

The respiratory motion of the liver is considerably complex (1) and especially large in the cranial-caudal (CC) direction (2). Interventional strategies for managing tumor motion such as respiratory-gating (3), breath-hold (4) and tumortracking radiotherapy (5) can reduce the increase in the

This article is freely accessible online.

Correspondence to: Yoshihiro Ueda, MSc, Department of Radiation Oncology, Osaka International Cancer Institute, 3-1-69 Otemae, Chuo-ku, 537-8567 Osaka, Japan. Tel: +81 669451181, Fax: +81 669451900, e-mail: uedetu@gmail.com

Key Words: SBRT, liver, 4DCT, setup accuracy. internal target volume (ITV) caused by respiratory motion to reduce the irradiated volume. Respiratory-gated radiotherapy manages respiratory motion as radiation is delivered during a specified phase of the respiratory cycle, especially the exhale phase in the case of liver cancer (6). Hence, accurate and precise target localization during the exhale phase is essential before each treatment session (7).

The target for liver cancer, however, unlike that for lung tumors, is difficult to visualize due to lack of contrast between tumor and normal tissue in on cone-beam computed tomography (CBCT), in-room CT-on-rail and X-ray fluoroscopic images (8), so that some surrogates, such as a bone structure, a diaphragm or fiducial markers, are used for target localization. Wunderink et al. (9) used registration based on bone landmarks, whereas Case et al. (10), Guckenberger et al. (11) and Eccles et al. (12) a diaphragmguided patient setup. Some investigators inserted fiducial markers near the tumor to improve image registration accuracy $(1,13)$. As reported by Seppenwoolde et al. (14), a comparison of bone anatomy, three-dimensional (3D) diaphragm dome and fiducial marker registration showed that fiducial marker registration was the most accurate.

However, there are some risks in using fiducial makers. First, fiducial markers are highly invasive, and extensive experience with percutaneous liver biopsies shows that the risk of complications, pain or bleeding is 1 to $4 \%$ (15). Second, there is a risk of tumor size change during the time required between the implantation of markers and the start of therapy. Last is the fiducial migration in organs. Kothary et al. (16) reported that in 6 of 139 patients, the fiducial makers migrated. Unrecognized migrations within the organs of interest lead to miss-targeting. Taking these results into account, evaluation of the accuracy of non-fiducial-based patient registration, such as diaphragm-guided registration, is thus urgently needed to avoid these risks.

Four-dimensional computed tomography (4DCT) has been used for treatment planning and target localization in gated 
radiotherapy $(6,8)$. Some investigators have reported on the unique artifacts of 4DCT. Yamamoto et al. (17) found that images of most cases seen in their department showed at least one artifact and found that these unique artifacts cannot be eliminated from 4DCT. Rieltzl et al. (18) reported that respiratory irregularities prevented correct imaging of a diaphragm with retrospective resorting software. We compared this accuracy with that of registration using light breath-holding CT images (BHCT), which are not affected by respiratory irregularities. No comparative data are available regarding the accuracy of two reference images for the surrogate-guided registration. This study attempted to fill this gap by investigating these two common CT images to evaluate the registration accuracy in respiratory-gated radiotherapy for liver cancer.

\section{Materials and Methods}

Subjects for the clinical case. Between May 2012 and April 2015, 35 patients underwent stereotactic body radiotherapy (SBRT) for liver cancer at our institution. Of those, 17 were enrolled in the present study, which was under the Institutional Review Board exempt status. Prior to treatment simulation, each patient was implanted percutaneously with one or two VISICOIL fiducial markers (diameter: $0.75 \mathrm{~mm}$, length: $5 \mathrm{~mm}$; Core Oncology, Santa Barbara, CA, USA) close to a tumor in the liver tissue under ultrasound guidance. Twelve patients were implanted with two and five patients with one fiducial marker. With the diaphragm-guided registration, all fiducial markers were implanted in the liver as targets. With the fiducial-guided registration of 12 cases, the fiducial marker on the cranial side was used as a surrogate and the one on the caudal side as a target.

For CT simulation, 4DCT by means of a CT scanner (LightSpeed 16 slices; GE Medical Systems, Waukesha, WI, USA) was administered to each patient under free breathing and immobilization with the BodyFix double-vacuum system (Elekta, Schwabmuenchen, Germany). Thus, BHCT was acquired in helical mode. The slice thickness and field of view (FOV) of these CT images were $2.5 \mathrm{~mm}$ and $50 \mathrm{~cm}$, respectively. The CT scanner was equipped with a real-time position management system (RPM) (Varian Medical Systems, Palo Alto, CA, USA) that acquired positions of infrared-reflective markers on the patient abdomen at a rate of 30 samples/s. RPM provided the respiratory signal to generate 10 respiratory phases of CT images based on phase-based sorting. During acquisition of the respiratory signal, displacements were only extracted during the actual liver scan and the minimum and maximum positions were identified in the signal for normalizing the displacements. With the normalized displacements, the standard deviation of an end-expiratory position in \% was defined as a respiratory irregularity because the abdominal position relative to RPM camera and the amplitude of the abdominal motion were different in each patient. The maximum intensity projectionCT (MIP; expiratory phase four-dimensional (Ex4D) CT) reconstructed from three bins $(40 \%, 50 \%$, and $60 \%$ phases) was used for radiotherapy planning and registration. The artifacts on sagittal images of 4DCT were identified by physicists as incomplete and overlapping as has been reported by Yamamoto et al. (17).

The gross tumor volume (GTV) was defined by phases CT of 40$60 \%$. To create the clinical target volume (CTV) a margin of $3 \mathrm{~mm}$

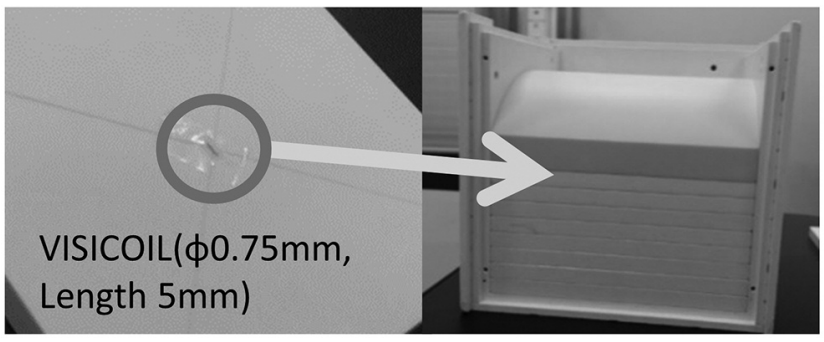

Figure 1. Dome phantom with a VISICOIL was inserted in a cubic, $18 \times 18 \times 18 \mathrm{~cm}^{3}$ (I'mRT phantom; IBA Dosimetry, Schwarzenbruck, Germany).

was added in all directions. To consider tumor motion into the dose calculation, the sum volume of the CTV in each phase of the CT images was defined as the internal target volume (ITV). An isotropic margin of $5 \mathrm{~mm}$ was then added to the ITV to form the PTV. Irradiation was delivered via seven non-coplanar photon beams of $6 \mathrm{MV}$ each. The total prescribed dose was $48 \mathrm{~Gy}$ at the isocenter and delivered in four fractions.

Phantom study to verify the performance of the registration with Ex$C B C T$. With this phantom study, the accuracy of the registration system with Ex-CBCT was summarized by means of the phantom study. The free-breathing CBCT (FB-CBCT) images, which were reconstructed from whole projections including full respiratory phases, showed significant motion artifacts of blurring on the diaphragm and fiducial markers. To remove these artifacts, expiratory phase (40\%-60\%) projections were extracted from whole projections using in-house software for reconstruction of expiratory phase CBCTs (Ex-CBCT). According to a study by Bedder (8), at the end of expiration (i.e., the $40 \%-60 \%$ phases) respiratory gating may be the most accurate. A phantom study was performed to evaluate the accuracy of the diaphragm-guided registration using Ex-CBCT. An on-board imaging system (OBI) mounted on a Varian 23EX linear accelerator (Varian Medical Systems, Palo Alto, CA, USA) was used for acquiring CBCTs in this study. The dome phantom (Figure 1), imitating the liver with a VISICOIL fiducial marker (Core Oncology) $0.75 \mathrm{~mm}$ in diameter and a length of 5 $\mathrm{mm}$, was moved with the Respiratory Platform Quasar (Modus Medical Devices, London, ON, Canada) in the CC direction with one of the following waveforms: cosine waveform, cosine fourthpower waveform or two types of combined waveforms (cosine waveform combined with either double the amount or half the amount of cosine fourth-power waveform). The experiment was performed in the $\mathrm{CC}$ direction only because the motion along the $\mathrm{CC}$ direction is significantly larger and is impacted by the radiation dose. Combined waveforms were assumed to be irregular in waveform. The dome edge was placed on the cranial side, $1.0 \mathrm{~cm}$ from the isocenter at the end of expiration. The peak-to-peak motion was $2.0 \mathrm{~cm}$ and respiratory cycles were $3.0,4.0$ and $5.0 \mathrm{~s}$ for each of the waveforms. Three CBCT images were then obtained for each of the moving patterns.

The algorithm to track dome motion on projections. The raw data consists of 670 projections for the reconstruction of CBCT. Tracking dome would be impossible using the normal template-matching technique because the vision of the dome is different with each 
A

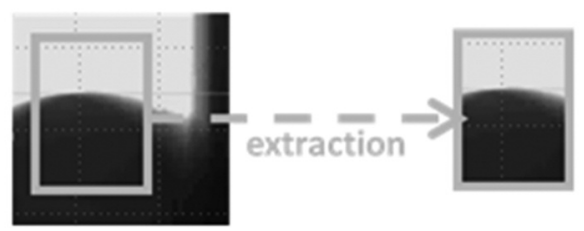

B N-1

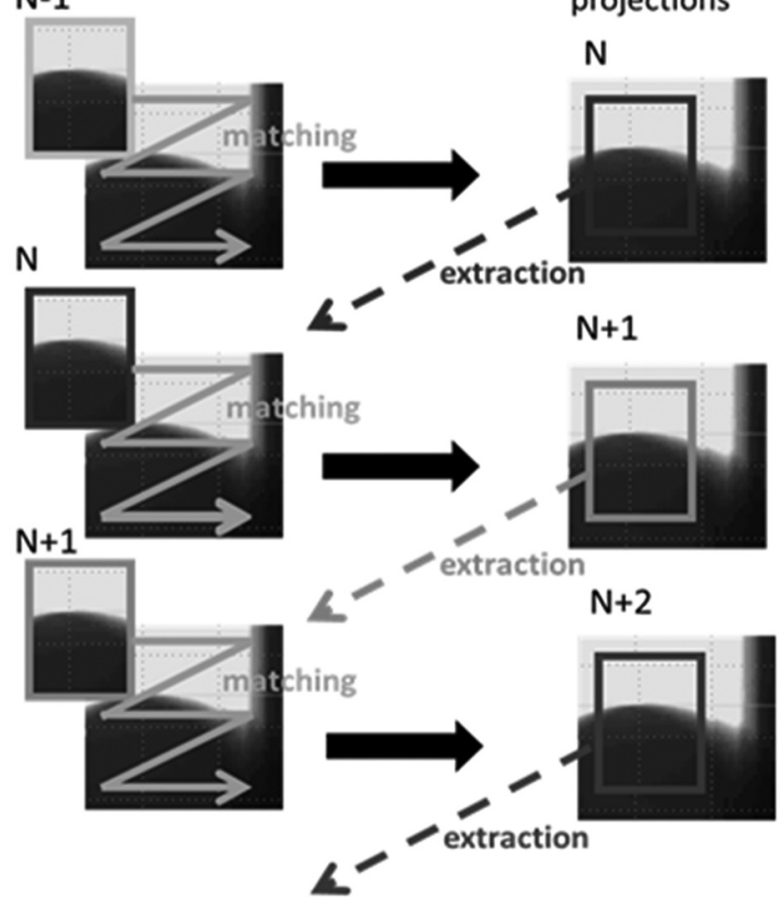

Figure 2. Schematic for the algorithm of the in-house software to detect a liver dome using a template matching technique. (A) The region extracted from the first projection used as the template image including the dome edge. (B) Matching of the images with the cone beam computed tomography $(C B C T)$ projections. The region of the $n^{\text {th }} C B C T$ projection overlapping with a given template image was detected and used as the template image for matching with the $(n+1)^{\text {th }} C B C T$ projection.

projection scanned at a different gantry angle. We improved the template-matching program to detect respiratory motion from the projections based on the assumption that adjacent projections were scanned at almost the same angle. This algorithm is described in Figure 2. The first template, which is the region including the dome, was defined in the first projection (Figure 2A). It was verified that the change of brightness for projections had no effect on dome tracking by the software. The region corresponding to the first template was searched on the second projection and used as the second template image for searching on the third projection. In this way, the region on the $\mathrm{n}^{\text {th }}$ projection identified by templatematching was used as the $\mathrm{n}^{\text {th }}$ template for matching on the $(\mathrm{n}+1)^{\text {th }}$ projection, as shown in Figure 2B. The motion of the dome was obtained by this algorithm along the cranial-caudal direction. The end inspiration, positioned at the upper edge on the most caudal side, was defined as the 0 phase, with $100 \%$ corresponding to a complete breathing cycle. The end expiration, positioned at the upper edge on the most caudal side, was defined as the $50 \%$ phase. The exhale projections (166 on average) within the $40-60 \%$ phase were transferred to the $\mathrm{CBCT}$ reconstruction software (Varian) to create the Ex-CBCT images with a slice thickness of $1.0 \mathrm{~mm}$ and field of view (FOV) of $45 \mathrm{~cm}$.

Registration methods for the phantom study and the clinical case. Planning CT images were acquired in the helical mode with a CT scanner (LightSpeed) while the dome phantom was stationary to represent the stationary liver. For these $\mathrm{CT}$ images, the slice thickness and FOV were $2.5 \mathrm{~mm}$ and $50 \mathrm{~cm}$, respectively. Each Ex-CBCT and the planning CT were registered in accordance with the dome and fiducial markers using registration software for the ARIA system (Varian Medical Systems). The first registration using the liver dome as a surrogate was performed in the lung field window, followed by the registration using a fiducial marker in the bone window to detect residual errors for surrogate-guided localization using the dome.

Before every treatment, free-breathing CBCT (FB-CBCT) was used for patient setup in the pelvis mode with an on-board imaging system $(\mathrm{OBI})$ mounted on a Varian 23EX Clinac ${ }^{\circledR}$ linear accelerator (Varian Medical Systems) in clinical case. To remove motion artifacts from FB-CBCT, expiratory phase (40\%, 50\%, and 60\%) projections were extracted from whole projections using an inhouse software for reconstruction of the expiratory phase CBCTs (Ex-CBCT). According to a study by Bedder et al. (8), respiratory gating at the end of expiration (i.e., the $40 \%-60 \%$ phases) appears to be the most precise. The expiratory projections (166 on average) within the $40 \%-60 \%$ phase were transferred retrospectively to CBCT reconstruction software (Varian Medical Systems) to create Ex-CBCT images with a slice thickness of $1.0 \mathrm{~mm}$ and FOV of 45 $\mathrm{cm}$. In 17 cases, $68 \mathrm{Ex}$-CBCT images sets were obtained. Figure $3 \mathrm{~A}$ shows coronal images for Ex-CBCT and $\mathrm{FB}-\mathrm{CBCT}$ in one case. The registrations were performed for the diaphragm registration and 48 registrations for the fiducial registration.

The surrogate registration methods are shown in Figure 3B. The diaphragm registration was performed in the lung field window determined by diaphragm domes viewed in three planes (transverse, coronal, and sagittal) including the diaphragm dome top. Fiducial registration was performed in the bone window on the cranial side of the fiducial marker viewed in three planes. The diaphragmguided registration errors (DRE) expressed in mm were defined as the differences between the diaphragm- and fiducial-guided registrations. The fiducial-guided registration errors (FRE) expressed in $\mathrm{mm}$ with the fiducial-guided registration were defined as the differences between upper and lower fiducial registrations. DRE and FRE were measured four times with daily CBCT in each case in three directions: left-right (LR), anterior-posterior (AP), and cranio-caudal (CC).

Data analysis. The group mean of DRE and FRE were calculated as the average for all cases. The systematic error $(\Sigma)$ and random error $(\sigma)$ of setup error components were defined as the SD of systematic errors and the root-mean-square of random errors, respectively. The absolute group mean errors of registration methods and reference images were compared. The errors were calculated independently in three directions-LR, AP, and CC -and 3D vector length was calculated from errors in the three directions. The correlations between absolute errors and surrogate-to-target distance and respiratory irregularity in all directions were also investigated. 

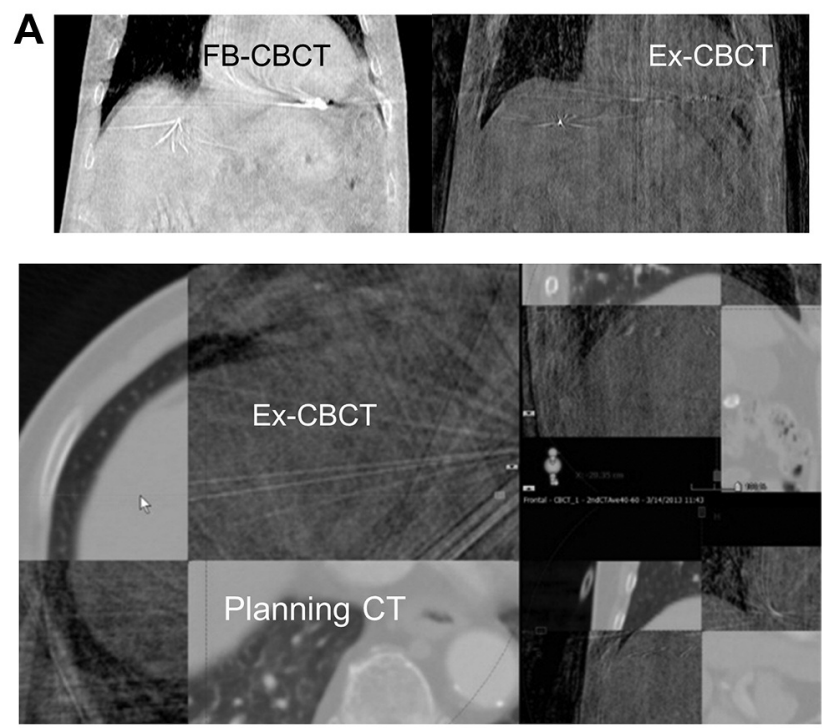

The diaphragm registration in lung field

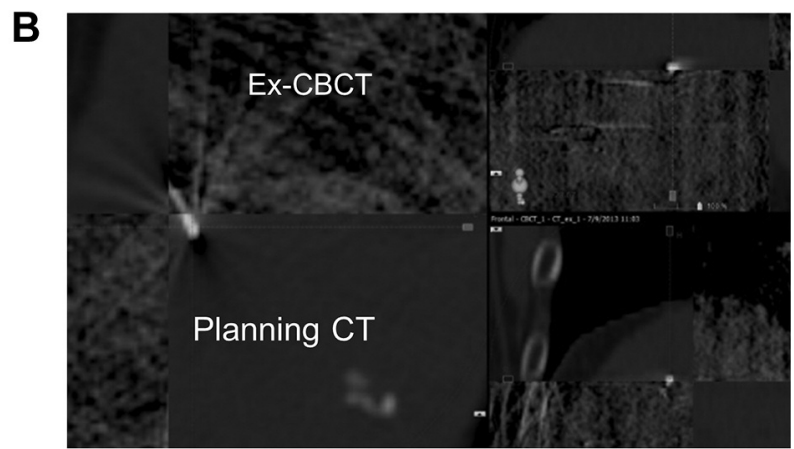

The fiducial maker registration in bone field

Figure 3. Expiratory phase cone beam computed tomography (Ex$C B C T)$ with diaphragm and fiducial registration for a clinical case. (A) $E x-C B C T$ and free breathing (FB)-CBCT. The diaphragm and fiducial marker are more clearly visible on Ex-CBCT than on FB-CBCT. (B) Diaphragm-guided registrations were performed in three planes in the lung field. (C) Fiducial-guided registrations were performed in three planes in the bone field.

For statistical analysis, a non-parametric Wilcoxon signed rank test was applied using SPSS ver. 16.0 (Chicago, IL, USA) to compare the accuracy of registration with BHCT and Ex4D. Differences were considered significant at $p<0.05$.

\section{Results}

Phantom study. Figure 4A shows a position detected on a projection by using a in-house software as a solid line and displacements for the moving dome are shown as a cosine waveform along the $\mathrm{CC}$ direction. The waveform was utilized for extracting projections during expiratory phases. Figure 4B shows two Ex-CBCT coronal images of a fiducial marker in the dome phantom moving along the cosine waveform. The left image was reconstructed from the $40-60 \%$ phase projections, and the right image from the $45-55 \%$ phases. The left Ex-CBCT image contained an artifact of blurring because the dome phantom was moving during 40-60\% along the cosine waveform. The fiducial marker edge in the $45-55 \%$ phase Ex-CBCT was sharper than that in the $40-60 \%$ phase. The upper edges represent the end-expiratory position in both images which was used for the fiducial-guided registration. Pixel values of the upper edges in the lung field for both the dome and the fiducial marker were greater so that retention time was longer during the end-expiratory phase. The accuracy of each waveform and respiratory cycle for the dome-guided registration was almost the same, with an overall mean of $0.0 \pm 0.2 \mathrm{~mm}$. In the phantom study, it was evident from these results that the upper edge in the dome and fiducialguided registration indicated the expiratory position with all waveforms.

Clinical case. The mean (range; min, max) maker-to-tumor distance was $4.3 \pm 2.3 \mathrm{~cm}(1.5,9.4)$. The mean (range; min, max) marker-to-marker distance was $4.1 \pm 2.5 \mathrm{~cm}(1.2,10.9)$, and diaphragm-to-marker distance was $9.7 \pm 9.7 \mathrm{~cm}(1.2$, 10.9 ) on BHCT in the $3 \mathrm{D}$ vector field. On $40 \%, 50 \%$, and 60\%-phase CT images, 12 patients $(71 \%$ of the total patients) had at least one artifact. The mean (range; min, $\max$ ) of the variation at end-expiratory positions on RPM respiratory signal while scanning the liver was $3.7 \% \pm 2.4 \%$ $(0.7 \%, 9.5 \%)$. In the case of some observed artifacts, the mean (range; $\min , \max$ ) of the variation was $5.0 \% \pm 2.4 \%$ $(1.7 \%, 9.5 \%)$ and without artifacts it was $2.0 \% \pm 0.8 \%(0.7 \%$, $3.0 \%$ ). The mean \pm SD (range) of the difference between the upper slice positions of the diaphragm on BHCT and Ex4D was $0.0 \pm 0.3 \mathrm{~cm}$, while the expiratory levels for the two modalities were almost the same.

Table I summarizes group mean errors, $\Sigma$ and $\sigma$ for setup errors of surrogate-guided registrations using Ex4D and $\mathrm{BHCT}$. In the $\mathrm{CC}$ direction, the systematic errors for DRE and FRE with Ex4D were larger than those with BHCT. In Figure 5A and B, the box plots of absolute DRE are represented with reference images of Ex4D and BHCT in the three directions and the 3D vector field. (A) was the case that upper VISICOIL was the target and (B) was the case that lower VISICOIL was the target in the diaphragm registration. For each box plot, the top bar indicates the maximum observation, while the lower bar indicates the minimum observation; the top of a box is the upper or third quartile, the bottom of a box is the lower or first quartile and the middle bar is the median value. In all directions, median and first quartile bars for BHCT were lower than those for Ex4D. The mean \pm SDs of the absolute DRE in LR, $\mathrm{AP}, \mathrm{CC}$ directions and $3 \mathrm{D}$ vector were $1.9 \pm 1.3,2.7 \pm 1.8$, 

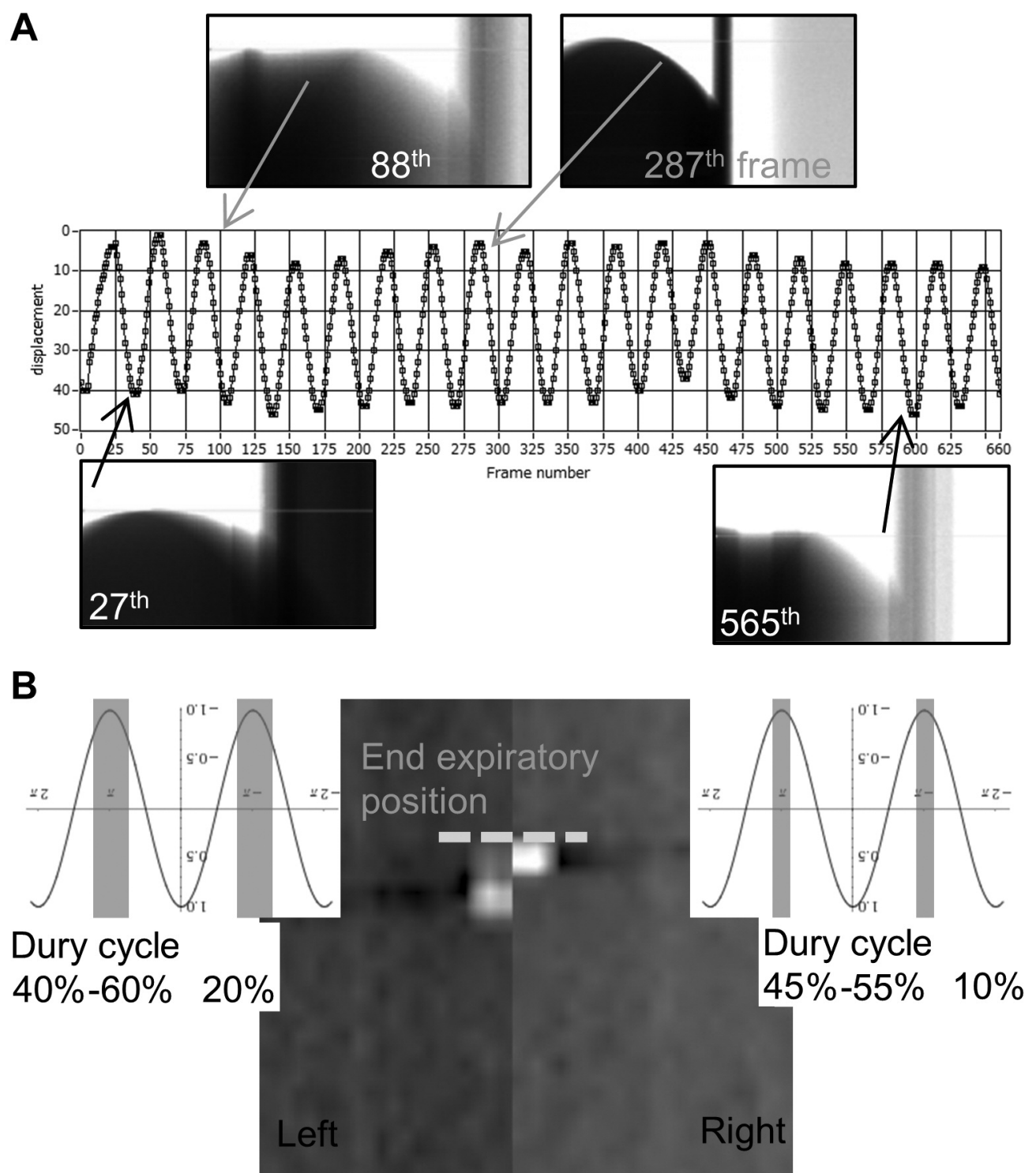

Figure 4. Maker position in expiratory phase cone beam computed tomography (Ex-CBCT) images at end expiratory phase. (A) A position detected with the in-house software is shown as a solid line on a projection and displacements for the moving dome are shown as a cosine waveform along the CC direction. The waveform was utilized for extracting projections during the expiratory phase. (B) Two Ex-CBCT coronal images of a fiducial marker moving along the cosine waveforms. The right image was reconstructed from the $45 \%-55 \%$ phase projections and the left image from the 40\%-60\% phases.

$2.6 \pm 1.7$ and $4.5 \pm 2.2 \mathrm{~mm}$ with Ex4D and $1.8 \pm 1.8,2.6 \pm 1.9$, $1.8 \pm 1.7$ and $4.1 \pm 2.3 \mathrm{~mm}$ with BHCT, respectively. The absolute DRE for Ex4D in the CC direction was significantly larger than that for BHCT $(p<0.01)$. In Figure $5 \mathrm{C}$, the box plots of absolute FRE for Ex4D and BHCT in the three directions and $3 \mathrm{D}$ vector are shown. In the $\mathrm{LR}$ direction, the absolute FRE for Ex4D and BHCT were almost the same. In the other directions, the median and first quartile bars for BHCT were lower than those for Ex4D. The mean \pm SD of the absolute FRE errors for LR,
$\mathrm{AP}, \mathrm{CC}$ directions and 3D were 1.2 $\pm 1.0,1.0 \pm 0.8,1.4 \pm 1.2$ and $2.4 \pm 1.2 \mathrm{~mm}$ with Ex4D, and $1.1 \pm 1.0,0.9 \pm 0.7,1.1 \pm 1.1$ and $2.1 \pm 1.3 \mathrm{~mm}$ for BHCT. There were no significant differences between Ex4D and BHCT in any direction $(p>0.64,0.52$, and 0.07).

Figures 6 and 7 show histograms of absolute DRE and FRE for Ex4D (a) and BHCT (b) in the three directions. In the CC direction, the peak value for Ex4D was larger than that for BHCT in DRE and FRE. The ratio of the absolute DRE with errors of more than $5.0 \mathrm{~mm}$ was $10.5 \%, 25.4 \%$, and $21.1 \%$ for 
Table I. Group mean errors (GM) and components ( $\Sigma$ and $\sigma)$ for setup errors of surrogate-guided registrations for each reference image.

\begin{tabular}{|c|c|c|c|c|c|}
\hline Surrogate & Reference images & Directions & GM & $\Sigma$ & $\sigma$ \\
\hline \multirow[t]{8}{*}{ Diaphragm } & 4DCT & LR & -0.1 & 2.2 & 1.5 \\
\hline & & $\mathrm{AP}$ & 1.1 & 2.8 & 1.9 \\
\hline & & $\mathrm{CC}$ & 0.9 & 2.8 & 1.6 \\
\hline & & $3 \mathrm{D}$ & 4.5 & 2.2 & 1.9 \\
\hline & BHCT & LR & 0.3 & 1.8 & 1.5 \\
\hline & & AP & 1 & 2.9 & 1.6 \\
\hline & & $\mathrm{CC}$ & 1.1 & 2.1 & 1.4 \\
\hline & & $3 \mathrm{D}$ & 4.1 & 2.3 & 2.1 \\
\hline \multirow[t]{8}{*}{ Fiducial } & 4DCT & LR & -0.5 & 1.4 & 1.0 \\
\hline & & $\mathrm{AP}$ & -0.4 & 1.1 & 1.1 \\
\hline & & $\mathrm{CC}$ & 0.4 & 1.8 & 1.0 \\
\hline & & $3 \mathrm{D}$ & 2.6 & 1.3 & 1.1 \\
\hline & BHCT & LR & -0.4 & 1.5 & 0.9 \\
\hline & & AP & -0.1 & 0.8 & 0.9 \\
\hline & & $\mathrm{CC}$ & 0.6 & 1.5 & 0.8 \\
\hline & & $3 \mathrm{D}$ & 2.2 & 1.3 & 0.8 \\
\hline
\end{tabular}

LR: Left-right; AP: anterior-posterior; CC: cranio-caudal; 3D: 3D vector; 4DCT: 4-dimensitional CT; BHCT: exhale breath-holding CT.

Ex4D and $10.4 \%, 20.9 \%$, and $17.4 \%$ for BHCT in LR, AP and $\mathrm{CC}$ directions. In $\mathrm{AP}$ and $\mathrm{CC}$ direction, the ratios for $\mathrm{BHCT}$ were smaller than those for Ex4D. The CTV to PTV margin of $5 \mathrm{~mm}$ was too small to compensate for set up errors in the diaphragm guided registration for Ex4D and BHCT in all directions. The ratio of the absolute DRE with errors of more than $10.0 \mathrm{~mm}$ was $0 \%, 0.9 \%$, and $6.1 \%$ for Ex4D and $0 \%$, $2.6 \%$, and $5.2 \%$ for BHCT in LR, AP and CC directions. The ratio of the absolute FRE with errors of more than $5.0 \mathrm{~mm}$ were $2.0 \%, 2.0 \%$, and $4.1 \%$ for Ex4D and $0 \%, 0 \%$, and $2.1 \%$ for BHCT in LR, AP, and CC directions.

The relationships between the absolute DRE for each reference image and the diaphragm-to-marker distance were determined in all directions. None of the reference images showed any correlation in any direction. The relationships between the absolute FRE for each reference image and the marker-to-marker distance were determined in all directions. The values for correlations (Rs) between the absolute FRE for Ex4D and the marker-to-marker distance were -0.12, 0.07, 0.74 and 0.70 in the RL, AP, CC, and 3D directions, respectively, with positive correlations in the $\mathrm{CC}$ and $3 \mathrm{D}$ directions. Corresponding Rs between the absolute FRE for BHCT and the marker-to-marker distance were $-0.21,-0.29,0.41$, and 0.33 , with weak positive correlations in the $\mathrm{CC}$ and $3 \mathrm{D}$ directions.

The relationships between the absolute DRE and FRE for Ex4D in the CC direction and 3D vector and the irregularities of expiratory positions are summarized in Figure 8 . Rs between irregularities and the absolute DRE were $0.0,0.0,0.65$ and 0.31 in the $\mathrm{LR}, \mathrm{AP}, \mathrm{CC}$, and $3 \mathrm{D}$ directions, respectively, with positive correlations in the $\mathrm{CC}$
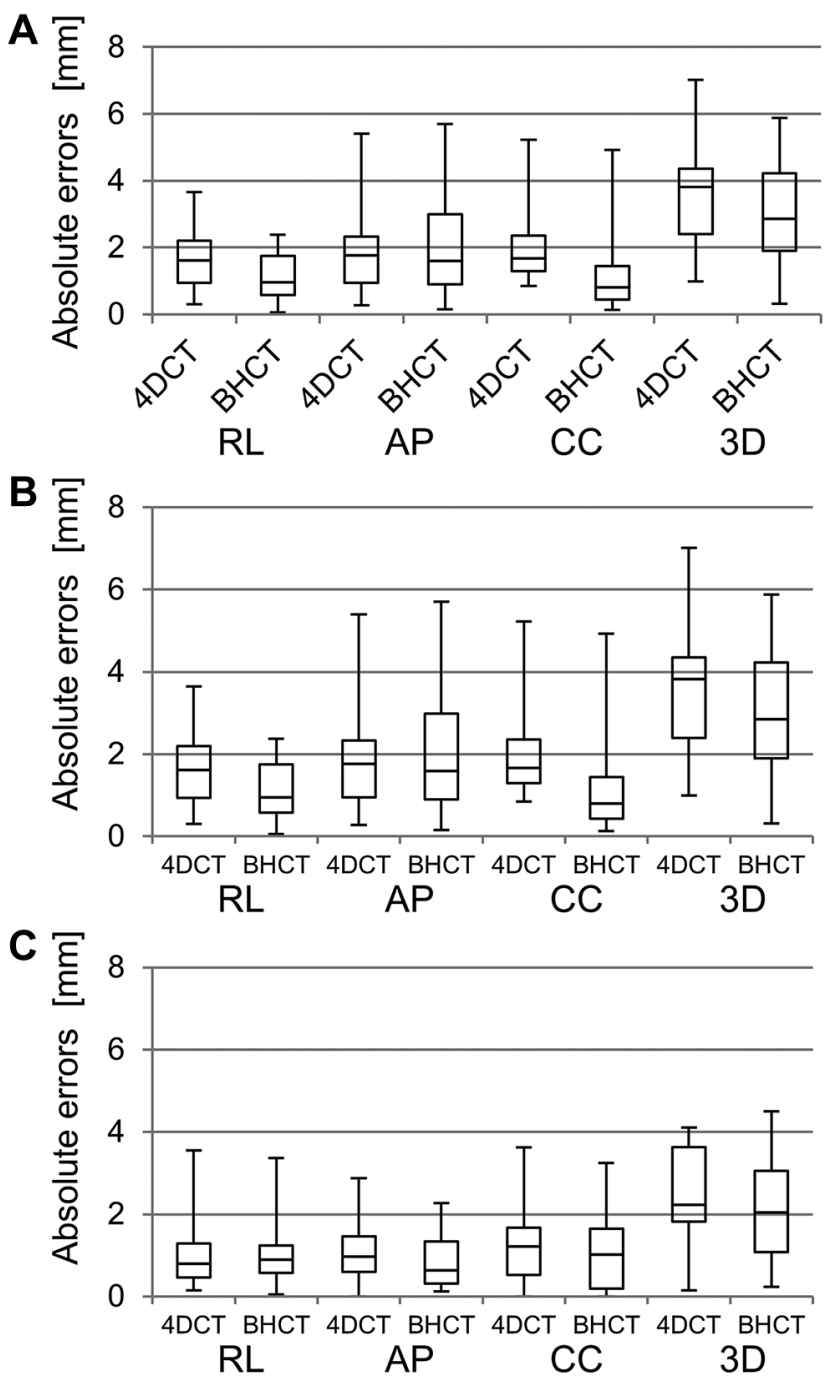

Figure 5. The box plots of absolute diaphragm-guided registration errors (DRE) to upper VISICOIL (A) and lower VISICOIL $(B)$. (C) The box plots of absolute fiducial-guided registration errors (FRE) in each of the four directions. Ex4D: Expiratory phase 4DCT; BHCT: breathholding computed tomography.

and 3D directions. Corresponding Rs between the absolute FRE and the irregularities were $0.36,0.12,0.54$ and 0.59 in the LR, AP, CC, and 3D directions.

In the case of observed artifacts, the mean $\pm \mathrm{SD}$ values of the absolute DRE were 1.8 $\pm 1.4,2.6 \pm 1.7,2.9 \pm 1.8$, and $4.6 \pm 2.4 \mathrm{~mm}$, and without artifacts they were $1.7 \pm 0.9,3.0 \pm 2.0,2.0 \pm 0.8$, and $4.3 \pm 2.0 \mathrm{~mm}$ in the LR, AP, CC, and 3D directions, respectively. In the $\mathrm{CC}$ and $3 \mathrm{D}$ directions in particular, the unique artifacts of Ex4D resulted in large absolute DRE. In the case of the fiducial-guided registration, the mean \pm SD of the absolute FRE with Ex4D and some observed artifacts were 1.5 $\pm 1.0,1.2 \pm 0.8$, $1.6 \pm 1.3$, and $3.0 \pm 1.2 \mathrm{~mm}$, and without artifacts they were $0.7 \pm 0.5,1.1 \pm 0.9,1.2 \pm 0.7$, and $1.71 .1 \mathrm{~mm}$ in the LR, AP, CC, 

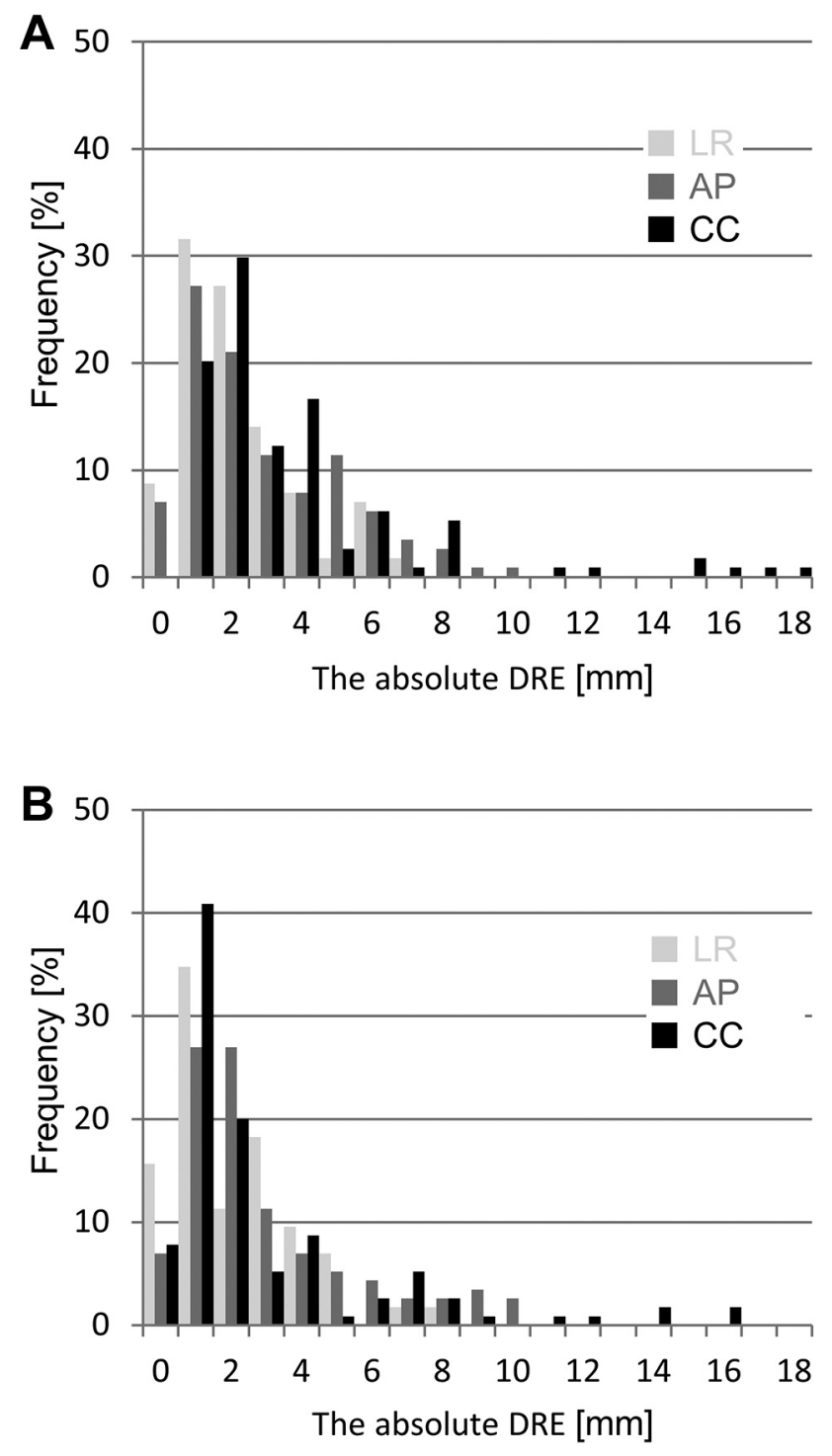

Figure 6. Histograms of the absolute diaphragm-guided registration errors (DRE) for expiratory phase $4 D C T(E x 4 D)(A)$ and breath holding computed tomography $(B H C T)(B)$ in three directions.

and 3D directions, respectively. In all directions, absolute FRE with Ex4D and some observed artifacts were larger with Ex4D without any observed artifacts. Table II also shows $\Sigma$ and $\sigma$ for setup errors of surrogate-guided registrations using Ex4D with and without artifacts. In LR and CC directions, $\Sigma$ s for DRE and FRE with Ex4D without artifacts were improved compared to those with artifact.

\section{Discussion}

4DCT scan is superior for detecting patient-specific motion and defining ITV (19). On the other hand, other recently
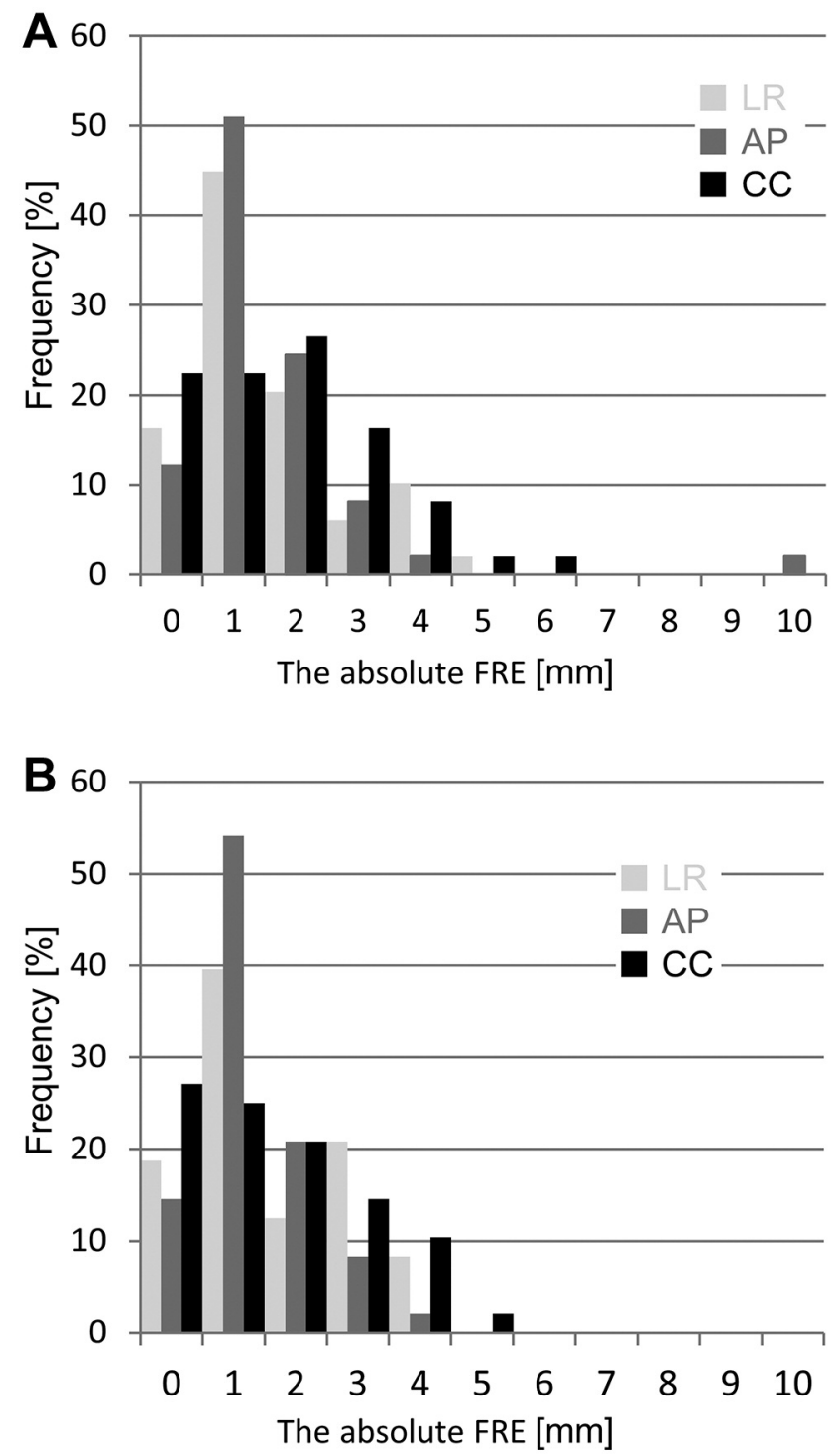

Figure 7. Histograms of the absolute fiducial-guided registration errors (FRE) for expiratory phase 4DCT (Ex4D) (A) and breath holding computed tomography $(B H C T)(B)$ in three directions.

published reports have questioned 4DCT's usefulness for defining ITV. Ge et al. (19) claimed that the motion in 4DCT could not adequately represent actual motion during treatment due to the irregularity of patient breathing. Yamamoto et al (17) and Rieltzel et al. (18) reported on the unique artifacts with 4DCT, with Yamamoto et al. finding that $95 \%$ of their cases showed at least one artifact on 4DCT images and pointing out that the usual version of this technique cannot remove artifacts resulting from respiratory motion of many patients. In this study, $71 \%$ of the cases showed at least one artifact in expiratory phase $\mathrm{CT}$ images. In addition, there was no artifact blurring because expiratory phase $\mathrm{CT}$ images were 

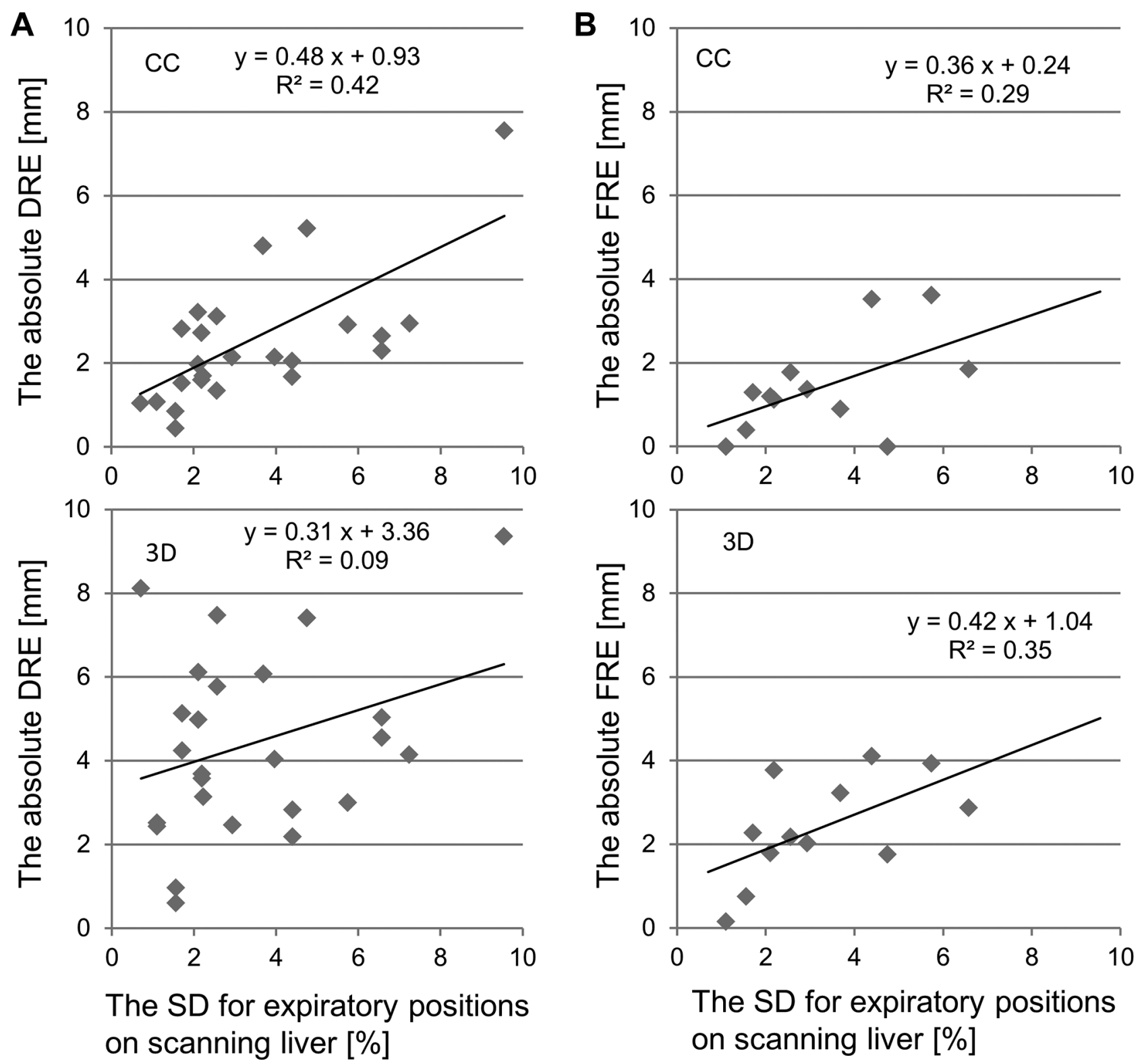

Figure 8. Relationships between standard deviations (SD) for expiratory positions during four-dimensional CT scanning and each of the absolute surrogate guided registration errors; (A) the diaphragm-guided registration errors (DRE) and (B) the fiducial-guided registration errors (FRE) for expiratory phase 4DCT (Ex4D).

acquired when the liver motion was stable. Specifically, a few blurring artifacts were observed in our study because liver motion was stable in the expiratory phase. For the reconstruction of 4DCT, $\mathrm{CT}$ images acquired at different positions and timing were sorted retrospectively. Respiratory irregularity during CT scanning causes deformation of the liver shape and results in large setup errors.

With the diaphragm-guided registration using Ex4D, the absolute DRE were significantly larger than those when BHCT was used in the $\mathrm{CC}$ direction. The registration error for Ex4D was large in comparison with the result reported by Seppenwoolde et al. (14). We found that the absolute prediction setup errors with the diaphragm- and fiducial-guided registration showed positive correlations $(\mathrm{R}=0.64$ and $\mathrm{R}=0.54$, respectively) with the respiration irregularity detected during 4DCT scanning in the CC direction (Figure 5). Using Ex4D with some observed artifacts, the absolute DRE and FRE were larger than those when Ex4D without artifacts was used. With 
Table II. Group mean errors (GM) and components ( $\Sigma$ and $\sigma)$ for setup errors of surrogate-guided registrations for expiratory phase 4DCT (Ex4D) with and without artifacts.

\begin{tabular}{lccrcc}
\hline Surrogate & Reference images & Directions & GM & $\Sigma$ & $\sigma$ \\
\hline \multirow{2}{*}{ Diaphragm } & Ex4D without & LR & -1.0 & 1.6 & 1.5 \\
& artifacts & AP & 1.5 & 3.4 & 1.9 \\
& & CC & 0.4 & 2.1 & 1.3 \\
& \multirow{4}{*}{ Ex4D with artifacts } & 3D & 4.3 & 2.0 & 2.1 \\
& & RL & 0.2 & 2.3 & 1.5 \\
& & AP & 0.9 & 2.6 & 1.9 \\
Fiducial & EC & 1.2 & 3.1 & 1.7 \\
& Ex without & 3D & 4.6 & 2.4 & 1.8 \\
& artifacts & LR & -0.1 & 1.0 & 0.8 \\
& & AP & -0.2 & 1.3 & 0.8 \\
& Ex4D with artifacts & CC & 0.0 & 1.5 & 0.9 \\
& & 3D & 1.7 & 1.1 & 0.8 \\
& & LP & -0.8 & 1.6 & 1.1 \\
& & CC & -0.5 & 1.0 & 1.2 \\
& & 3D & 3.0 & 1.8 & 1.0 \\
& & & & 1.2 & 1.2 \\
\hline
\end{tabular}

LR: Left-right; AP: anterior-posterior; CC: cranio-caudal; 3D: 3D vector; 4DCT: 4-dimensitional CT; BHCT: exhale breath-holding CT.

the diaphragm-guided registration, the largest difference was $1.5 \mathrm{~mm}$ in the $\mathrm{CC}$ direction, and with the fiducial-guided registration, FRE for Ex4D without any observed artifact were larger than those for Ex4D with some artifacts in all directions. In the absence of any observed artifacts, the SD for expiratory positions ranged from $0.7 \%$ to $3.0 \%$. When $4 \mathrm{DCT}$ with respiratory irregularity of less than $3 \%$ was used, the diaphragm-guided registration errors were almost the same as those when BHCT was used. For the surrogate-guided registration using Ex4D, the SD of expiratory positions should therefore be less than 3\% during CT scanning.

A limitation of this study was that the evaluation of registration with surrogate makers was demonstrated with fiducial makers instead of tumors. Seppenwoolde et al. (14) measured FRE using contrasted tumors on CT images and also maker-to-maker differences. In the fiducial maker registration, systematic errors for the targeted maker were smaller than those for tumor position in the CC and LR directions. Thus, in our study, FRE might have been smaller than the set-up errors for tumors if the surrogate-guided registration was performed to tumors. The migration of fiducial makers was another limitation of this study. Kothary et al. (16) evaluated the migration of fiducial makers in the lung, pancreas, and liver. There was no migration in the liver although fiducials migrated in the lung and pancreas in $9.1 \%$ and $3.3 \%$ of all the cases. Therefore, influence of the migration on the results of the study was small.

Again, in contrast to the results reported by Seppenwoolde et al. (14), the targeting accuracy with the fiducial-guided registration depended on the distance between the surrogates and tumors, whereas the targeting accuracy with the diaphragm-guided registration did not depend on the distance in our study. According to their report, for a marker-to-tumor distance of $<\sim 8.0 \mathrm{~cm}$, the systematic error SDs of the markerguided setup were significantly smaller in the CC and AP directions than for the diaphragm-based setup strategies. In our study, for a marker-to-marker distance of $<6.0 \mathrm{~cm}$ in the 3D vector or of $<\sim 4.0 \mathrm{~cm}$ in the $\mathrm{CC}$ direction, the absolute mean shifts were smaller than those with the diaphragm-guided registration. As pointed out by Park et al. (1), inter- and intrafractional variations in motion occur as the distance between the markers in the liver increases. These findings indicate that the fiducial marker must be located near the target.

\section{Conclusion}

In the surrogate-guided registration, BHCT improved the registration accuracy compared with Ex4D. Respiratory irregularity caused larger setup errors for surrogate-guided registration for Ex4D. To avoid unnecessary setup errors with 4DCT, the change in expiratory position during scanning should be less than $3 \%$.

\section{Conflicts of Interest}

The Authors have no conflicts of interest to declare in relation to this study.

\section{Authors' Contributions}

All the Authors participated in the writing and revision of this article and take responsibility for its content. The Authors confirm that the content of the manuscript has not been published, or submitted for publication elsewhere.

\section{Acknowledgements}

This study was supported by Health and Labour Sciences Research Grants for Promotion of Cancer Control Programs (H26-Cancer Policy-General-014) and JSPS KAKENHI Grant (Grant-in-Aid for Scientific Research (B) 15H04913).

\section{References}

1 Park JC, Park SH, Kim JH, Yoon SM, Song SY, Liu Z, Song B, Kauweloa K, Webster MJ, Sandhu A, Mell LK, Jiang SB, Mundt AJ and Song WY: Liver motion during cone beam computed tomography guided stereotactic body radiation therapy. Med Phys 39(10): 6431-6442, 2012. PMID: 23039678. DOI: $10.1118 / 1.4754658$

2 Balter JM, Dawson LA, Kazanjian S, McGinn C, Brock KK, Lawrence $\mathrm{T}$ and Ten Haken R: Determination of ventilatory liver movement via radiographic evaluation of diaphragm position. Int J Radiat Oncol Biol Phys 51(1): 267-270, 2001. PMID: 11516877. DOI: 10.1016/s0360-3016(01)01649-2 
3 Vedam SS, Kini VR, Keall PJ, Ramakrishnan V, Mostafavi H and Mohan R: Quantifying the predictability of diaphragm motion during respiration with a noninvasive external marker. Med Phys 30(4): 505-513, 2003. PMID: 12722802. DOI: 10.1118/1.1558675

4 Fracchiolla F, Dionisi F, Righetto R, Widesott L, Giacomelli I, Cartechini G, Farace P, Bertolini M, Amichetti M and Schwarz M: Clinical implementation of pencil beam scanning proton therapy for liver cancer with forced deep expiration breath hold. Radiother Oncol 154: 137-144, 2021. PMID: 32976870. DOI: 10.1016/j.radonc.2020.09.035

5 Poulsen PR, Murtaza G, Worm ES, Ravkilde T, O'Brien R, Grau C, Høyer M and Keall P: Simulated multileaf collimator tracking for stereotactic liver radiotherapy guided by kilovoltage intrafraction monitoring: Dosimetric gain and target overdose trends. Radiother Oncol 144: 93-100, 2020. PMID: 31786423. DOI: $10.1016 /$ j.radonc.2019.11.008

6 Gabryś D, Kulik R, Trela K and Ślosarek K: Dosimetric comparison of liver tumour radiotherapy in all respiratory phases and in one phase using 4DCT. Radiother Oncol 100(3): 360-364, 2011. PMID: 21974916. DOI: 10.1016/j.radonc.2011.09.006

7 Oshiro Y, Okumura T, Ishida M, Sugahara S, Mizumoto M, Hashimoto T, Yasuoka K, Tsuboi K, Sakae T and Sakurai H: Displacement of hepatic tumor at time to exposure in end-expiratorytriggered-pulse proton therapy. Radiother Oncol 99(2): 124-130, 2011. PMID: 21620501. DOI: 10.1016/j.radonc.2011.05.009

8 Beddar AS, Briere TM, Balter P, Pan T, Tolani N, Ng C, Szklaruk J and Krishnan S: 4D-CT imaging with synchronized intravenous contrast injection to improve delineation of liver tumors for treatment planning. Radiother Oncol 87(3): 445-448, 2008. PMID: 18194819. DOI: 10.1016/j.radonc.2007.12.009

9 Wunderink W, Méndez Romero A, Vásquez Osorio EM, de Boer HC, Brandwijk RP, Levendag PC and Heijmen BJ: Target coverage in image-guided stereotactic body radiotherapy of liver tumors. Int J Radiat Oncol Biol Phys 68(1): 282-290, 2007. PMID: 17448881. DOI: 10.1016/j.ijrobp.2006.12.034

10 Case RB, Sonke JJ, Moseley DJ, Kim J, Brock KK and Dawson LA: Inter- and intrafraction variability in liver position in nonbreath-hold stereotactic body radiotherapy. Int J Radiat Oncol Biol Phys 75(1): 302-308, 2009. PMID: 19628342. DOI: 10.1016/j.ijrobp.2009.03.058

11 Guckenberger M, Sweeney RA, Wilbert J, Krieger T, Richter A, Baier K, Mueller G, Sauer O and Flentje M: Image-guided radiotherapy for liver cancer using respiratory-correlated computed tomography and cone-beam computed tomography. Int J Radiat Oncol Biol Phys 71(1): 297-304, 2008. PMID: 18406894. DOI: 10.1016/j.ijrobp.2008.01.005
12 Eccles CL, Dawson LA, Moseley JL and Brock KK: Interfraction liver shape variability and impact on GTV position during liver stereotactic radiotherapy using abdominal compression. Int J Radiat Oncol Biol Phys 80(3): 938-946, 2011. PMID: 20947263. DOI: 10.1016/j.ijrobp.2010.08.003

13 Shirato H, Harada T, Harabayashi T, Hida K, Endo H, Kitamura K, Onimaru R, Yamazaki K, Kurauchi N, Shimizu T, Shinohara N, Matsushita M, Dosaka-Akita H and Miyasaka K: Feasibility of insertion/implantation of 2.0-mm-diameter gold internal fiducial markers for precise setup and real-time tumor tracking in radiotherapy. Int J Radiat Oncol Biol Phys 56(1): 240-247, 2003. PMID: 12694845. DOI: 10.1016/s0360-3016(03)00076-2

14 Seppenwoolde Y, Wunderink W, Wunderink-van Veen SR, Storchi P, Méndez Romero A and Heijmen BJ: Treatment precision of image-guided liver SBRT using implanted fiducial markers depends on marker-tumour distance. Phys Med Biol 56(17): 5445-5468, 2011. PMID: 21813963. DOI: 10.1088/ 0031-9155/56/17/001

15 Pawa S, Ehrinpreis M, Mutchnick M, Janisse J, Dhar R and Siddiqui FA: Percutaneous liver biopsy is safe in chronic hepatitis $\mathrm{C}$ patients with end-stage renal disease. Clin Gastroenterol Hepatol 5(11): 1316-1320, 2007. PMID: 17904916. DOI: 10.1016/j.cgh.2007.07.010

16 Kothary N, Heit JJ, Louie JD, Kuo WT, Loo BW Jr, Koong A, Chang DT, Hovsepian D, Sze DY and Hofmann LV: Safety and efficacy of percutaneous fiducial marker implantation for imageguided radiation therapy. J Vasc Interv Radiol 20(2): 235-239, 2009. PMID: 19019700. DOI: 10.1016/j.jvir.2008.09.026

17 Yamamoto T, Langner U, Loo BW Jr, Shen J and Keall PJ: Retrospective analysis of artifacts in four-dimensional CT images of 50 abdominal and thoracic radiotherapy patients. Int J Radiat Oncol Biol Phys 72(4): 1250-1258, 2008. PMID: 18823717. DOI: 10.1016/j.jijrobp.2008.06.1937

18 Rietzel E, Pan T and Chen GT: Four-dimensional computed tomography: image formation and clinical protocol. Med Phys 32(4): 874-889, 2005. PMID: 15895570. DOI: 10.1118/1.1869852

19 Ge J, Santanam L, Noel C and Parikh PJ: Planning 4dimensional computed tomography (4DCT) cannot adequately represent daily intrafractional motion of abdominal tumors. Int J Radiat Oncol Biol Phys 85(4): 999-1005, 2013. PMID: 23102840. DOI: 10.1016/j.ijrobp.2012.09.014

Received April 3, 2021

Revised May 2, 2021

Accepted May 6, 2021 